\begin{tabular}{|c|l|}
\hline Title & New description of the four-body breakup reaction \\
\hline Author(s) & Matsumoto, Takuma; Katō , Kiyoshi; Y ahiro, Masanobu \\
\hline Citation & $\begin{array}{l}\text { Physical Review C, 82(5), 051602-1-051602-5 } \\
\text { https://doi.org/10.1103/PhysRevC.82.051602 }\end{array}$ \\
\hline Issue Date & 2010 \\
\hline Doc URL & http://hdl.handle.net/2115/46853 \\
\hline Rights & ○ 2010 A merican Physical Society \\
\hline Type & article \\
\hline File Information & PhysRevC.82.051602.pdf \\
\hline
\end{tabular}

Instructions for use 


\title{
New description of the four-body breakup reaction
}

\author{
Takuma Matsumoto, ${ }^{1, *}$ Kiyoshi Katōo, ${ }^{2, \dagger}$ and Masanobu Yahiro ${ }^{3, \ddagger}$ \\ ${ }^{1}$ Meme Media Laboratory, Hokkaido University, Sapporo 060-8628, Japan \\ ${ }^{2}$ Division of Physics, Graduate School of Science, Hokkaido University, Sapporo 060-0810, Japan \\ ${ }^{3}$ Department of Physics, Kyushu University, Fukuoka 812-8581, Japan
}

(Received 2 June 2010; revised manuscript received 7 October 2010; published 9 November 2010)

\begin{abstract}
We present a novel method of smoothing discrete breakup cross sections calculated by the method of continuumdiscretized coupled channels. The smoothing method based on the complex scaling method is tested with success for a ${ }^{58} \mathrm{Ni}(d, p n)$ reaction at $80 \mathrm{MeV}$ as an example of three-body breakup reactions and applied to a ${ }^{12} \mathrm{C}\left({ }^{6} \mathrm{He}, n n{ }^{4} \mathrm{He}\right)$ reaction at $229.8 \mathrm{MeV}$ as an example of four-body breakup reactions. Fast convergence of the breakup cross section with respect to extending the model space is confirmed. The method is also applied to ${ }^{12} \mathrm{C}\left({ }^{6} \mathrm{He}, n n{ }^{4} \mathrm{He}\right)$ and ${ }^{208} \mathrm{~Pb}\left({ }^{6} \mathrm{He}, n n{ }^{4} \mathrm{He}\right)$ reactions at $240 \mathrm{MeV} / A$ and compared with the experimental data.
\end{abstract}

DOI: 10.1103/PhysRevC.82.051602

PACS number(s): 24.10.Eq, 25.60.Gc, 25.70.De

Exploring unstable nuclei far from the stable line is one of the most important subjects in nuclear physics. The unstable nuclei have exotic properties such as the halo structure [1-3] and the island of inversion [4]. As a feature of reactions induced by unstable nuclei, the projectile easily breaks up into its constituents. One of the most reliable methods for treating the projectile breakup processes over a wide range of incident energies is the continuum-discretized coupled channels (CDCC) method [5,6]. In CDCC, the scattering wave function of the total system is expanded with a finite number of bound and discretized continuum states of the projectile. The space spanned by these states is called the model space. The $S$-matrix elements calculated with CDCC converge as the model space is extended $[7,8]$. The converged CDCC solution is the unperturbed solution of the distorted Faddeev equations, and corrections to the solution are negligible within the spatial region in which the breakup processes take place $[9,10]$.

For scattering of a two-body projectile, the continuum states are classified by linear and angular momenta $k$ and $l$, respectively, between the two constituents. In CDCC, these momenta are taken up to upper limits, the $k$ continuum is divided into small bins, and the continuum states in each bin are averaged into a single state. This discretization procedure is called the average (Av) method. The Av method has been widely used, but its application has been limited to three-body breakup reactions as we will show. An alternative to the $\mathrm{Av}$ method is the pseudostate (PS) method [11-16], in which the continuum states $\left\{\psi^{(-)}(\boldsymbol{k})\right\}$ are replaced by pseudostates $\left\{\Phi_{n}\right\}$ obtained by diagonalizing the internal Hamiltonian of the projectile in a space spanned by $L^{2}$-type basis functions. One can adopt the transformed harmonic oscillator (THO) [11] or the Gaussian $[12,13]$ as the $L^{2}$-type basis functions. The validity of the PS method was confirmed for scattering of twobody projectiles by the agreement between CDCC solutions calculated with the two discretization methods [12-15].

\footnotetext{
*tmatsumoto@nucl.sci.hokudai.ac.jp

${ }^{\dagger}$ kato@nucl.sci.hokudai.ac.jp

${ }^{\ddagger}$ yahiro@phys.kyushu-u.ac.jp
}

For scattering of ${ }^{6} \mathrm{He}$ as a typical example of four-body breakup reactions, CDCC with the PS method based on Gaussian [14,15] or THO [16] basis functions was successful in describing the elastic scattering at not only high energies but also low energies near the Coulomb barrier. Thus, the back-coupling effect of four-body breakup processes on the elastic scattering is well described by the PS method.

For the three-body projectile, continuum wave functions $\psi^{(-)}(\boldsymbol{k}, \boldsymbol{p})$ are classified by momenta $(\boldsymbol{k}, \boldsymbol{p})$ conjugate to two internal coordinates $(\boldsymbol{y}, \boldsymbol{r})$ of the three-body system. The breakup $S$-matrix elements calculated with CDCC, $S_{n}$, are discrete in $\boldsymbol{k}$ and $\boldsymbol{p}$, although the exact ones $S(\boldsymbol{k}, \boldsymbol{p})$ are continuous. Thus, one needs a way of smoothing $S_{n}$. In principle this is possible by calculating the smoothing factor $\left\langle\psi^{(-)}(\boldsymbol{k}, \boldsymbol{p}) \mid \Phi_{n}\right\rangle[12]$, but in practice it is not easy, because evaluating $\psi^{(-)}(\boldsymbol{k}, \boldsymbol{p})$ for many combinations of $\boldsymbol{k}$, $p$, and $n$ is quite time consuming. Recently, we proposed two methods of obtaining the smoothing factor: one with a direct numerical integration [17] and another [18] with the complex scaling method (CSM) [19]. However, these require complicated numerical calculations, so that the convergence of the smoothed breakup cross section with respect to increasing the model space is not sufficient, particularly for the differential breakup cross section as a function of the excitation energy $\varepsilon$ of projectile $d \sigma / d \varepsilon$. If the Av method is applied with small momentum or energy bins, one can obtain $d \sigma / d \varepsilon$ without evaluating the smoothing factor. This was done with the hyperradial continuum wave function [20], but the convergence of the CDCC solutions has not been obtained yet for $d \sigma / d \varepsilon$ [21]. A way of circumventing these difficulties is to construct a method of obtaining the smoothing factor without evaluating $\psi^{(-)}(\boldsymbol{k}, \boldsymbol{p})$.

In this Rapid Communication, using CSM and CDCC, we propose a practical method of obtaining $d \sigma / d \varepsilon$ as a continuous function of $\varepsilon$ without evaluating $\psi^{(-)}(\boldsymbol{k}, \boldsymbol{p})$. CSM is a powerful tool for obtaining many-body resonance and weakly bound states [22]. Recently, it was applied to the electromagnetic transition of the core + nucleon + nucleon system, such as ${ }^{6} \mathrm{He}$ and ${ }^{11} \mathrm{Li}$ from the ground state to the continuum state with $\varepsilon$ [23,24]. CSM is applicable not only for resonances but also for continuum states, so that the 
transition strength is obtained as a continuous function of $\varepsilon$. The smoothing method proposed here is an alternative to the direct calculation of the smoothing factor $\left\langle\psi^{(-)}(\boldsymbol{k}, \boldsymbol{p}) \mid \Phi_{n}\right\rangle$ with the hyperradial continuum wave function $[25,26]$. The former is considered to be more practical than the latter, because the former does not require one to evaluate the continuum states $\psi^{(-)}(\boldsymbol{k}, \boldsymbol{p})$ for many combinations of $\boldsymbol{k}$ and $\boldsymbol{p}$. The validity of the new method is tested for a three-body breakup reaction, ${ }^{58} \mathrm{Ni}(d, p n)$ at $80 \mathrm{MeV}$, in which the "exact" breakup cross section is obtainable by calculating the smoothing factor with the direct numerical integration. The new method is applied to the ${ }^{12} \mathrm{C}\left({ }^{6} \mathrm{He}, n n^{4} \mathrm{He}\right)$ reaction at $229.8 \mathrm{MeV}$. A merit of the present smoothing method is that one can see fast convergence of the calculated breakup cross section with respect to extending the model space. The method is also applied to ${ }^{12} \mathrm{C}\left({ }^{6} \mathrm{He}, n n{ }^{4} \mathrm{He}\right)$ and ${ }^{208} \mathrm{~Pb}\left({ }^{6} \mathrm{He}, n n{ }^{4} \mathrm{He}\right)$ reactions at $240 \mathrm{MeV} / A$ and compared with the experimental data. In principle, this method is applicable not only for four-body breakup reactions but also for many-body breakup reactions.

We consider scattering of a projectile $B$ from a target $A$, in which $B$ is composed of three constituents $(x=b, c$, and $d$ ). The scattering is described by the four-body Schrödinger equation

$$
\left(H-E_{\text {tot }}\right)\left|\Psi^{(+)}\right\rangle=0
$$

with the outgoing boundary condition, where the total energy $E_{\text {tot }}$ satisfies $E_{\text {tot }}=E_{\text {in }}^{\mathrm{CM}}+\varepsilon_{0}$ for the corresponding incident energy $E_{\mathrm{in}}^{\mathrm{CM}}$ in the center of mass of the $B+A$ system and the ground-state energy $\varepsilon_{0}$ of $B$. The total Hamiltonian $H$ of this system is defined by

$$
H=K_{R}+U+H_{B}
$$

with

$$
\begin{aligned}
U & =U_{b}+U_{c}+U_{d}+V_{b}^{\text {Coul }}+V_{c}^{\text {Coul }}+V_{d}^{\text {Coul }}, \\
H_{B} & =K_{y}+K_{r}+V_{b c}+V_{c d}+V_{d b},
\end{aligned}
$$

where $H_{B}$ is an internal Hamiltonian of $B$. The relative coordinate between $B$ and $A$ is denoted by $\boldsymbol{R}$, and the internal coordinates of $B$ are denoted by a set of Jacobi coordinates $\boldsymbol{\xi}=(\boldsymbol{y}, \boldsymbol{r})$. Momenta conjugate to coordinates $\boldsymbol{R}$ and $(\boldsymbol{y}, \boldsymbol{r})$ are represented by $\boldsymbol{P}$ and $(\boldsymbol{p}, \boldsymbol{k})$, respectively. The kinetic energy operator associated with $\boldsymbol{R}(\boldsymbol{\xi})$ is represented by $K_{R}\left(K_{\xi}\right), V_{x x^{\prime}}$ is a nuclear-plus-Coulomb interaction between $x$ and $x^{\prime}$, and $U_{x}$ and $V_{x}^{\text {Coul }}$ are nuclear and Coulomb potentials between $x$ and $A$, respectively.

In CDCC with the pseudostate discretization method, the scattering is assumed to take place in a model space [12-15]:

$$
\mathcal{P}=\sum_{n}\left|\Phi_{n}\right\rangle\left\langle\Phi_{n}\right|,
$$

where $\Phi_{n}$ is an $n$th eigenstate obtained by diagonalizing $H_{B}$ with $L^{2}$-type basis functions. For simplicity, $B$ is assumed to have only one bound state $\Phi_{0}$. The four-body Schrödinger equation is then solved in the model space:

$$
\mathcal{P}\left(H-E_{\text {tot }}\right) \mathcal{P}\left|\Psi_{\text {CDCC }}^{(+)}\right\rangle=0 .
$$

The model space assumption has already been justified by the fact that calculated elastic and breakup cross sections converge with respect to extending the model space [12-15].
The exact $T$-matrix element to a breakup state with $(\boldsymbol{p}, \boldsymbol{k})$ can be described by

$$
T_{\varepsilon}(\boldsymbol{p}, \boldsymbol{k}, \boldsymbol{P})=\left\langle\psi_{\varepsilon}^{(-)}(\boldsymbol{p}, \boldsymbol{k}) \chi_{\varepsilon}^{(-)}(\boldsymbol{P})\left|U-V_{B}^{\mathrm{Coul}}\right| \Psi^{(+)}\right\rangle,
$$

where $V_{B}^{\text {Coul }}$ is a sum of Coulomb interactions between $B$ and $A$, but the arguments are replaced by $R$ :

$$
V_{B}^{\text {Coul }}(R)=V_{b}^{\text {Coul }}(R)+V_{c}^{\text {Coul }}(R)+V_{d}^{\text {Coul }}(R) .
$$

The final-state wave functions $\left|\psi_{\varepsilon}^{(-)}(\boldsymbol{p}, \boldsymbol{k})\right\rangle$ and $\left|\chi_{\varepsilon}^{(-)}(\boldsymbol{P})\right\rangle$ with the incoming boundary condition are defined by

$$
\begin{aligned}
{\left[T_{R}+V_{B}^{\text {Coul }}(R)-\left(E_{\mathrm{tot}}-\varepsilon\right)\right]\left|\chi_{\varepsilon}^{(-)}(\boldsymbol{P})\right\rangle } & =0, \\
\left(H_{B}-\varepsilon\right)\left|\psi_{\varepsilon}^{(-)}(\boldsymbol{p}, \boldsymbol{k})\right\rangle & =0,
\end{aligned}
$$

where $\quad E_{\text {tot }}-\varepsilon=(\hbar P)^{2} /\left(2 \mu_{R}\right) \quad$ and $\quad \varepsilon=(\hbar p)^{2} /\left(2 \mu_{y}\right)+$ $(\hbar k)^{2} /\left(2 \mu_{r}\right)$ for reduced masses $\mu_{R}$ and $\mu_{\xi}$ of coordinates $\boldsymbol{R}$ and $\boldsymbol{\xi}$, respectively. Inserting the approximately complete set, Eq. (5), into Eq. (7), we can find [12-15] that the $T$-matrix element is well approximated by

$$
T_{\varepsilon}(\boldsymbol{p}, \boldsymbol{k}, \boldsymbol{P}) \approx \sum_{n \neq 0}\left\langle\psi_{\varepsilon}^{(-)}(\boldsymbol{p}, \boldsymbol{k}) \mid \Phi_{n}\right\rangle T_{n}
$$

with the CDCC $T$-matrix element

$$
T_{n}=\left\langle\Phi_{n} \chi_{\varepsilon_{n}}^{(-)}\left(\boldsymbol{P}_{n}\right)\left|U-V_{B}^{\text {Coul }}\right| \Psi_{\mathrm{CDCC}}^{(+)}\right\rangle
$$

to an $n$th discrete breakup state $\Phi_{n}$ with an eigenenergy $\varepsilon_{n}$. Here Eq. (11) is derived by replacing $\boldsymbol{P}$ by $\boldsymbol{P}_{n}$ in $\chi_{\varepsilon}^{(-)}(\boldsymbol{P})$. $T_{n}$ is obtainable by CDCC, but it is quite hard to calculate the smoothing factor $\left\langle\psi_{\varepsilon}^{(-)}(\boldsymbol{p}, \boldsymbol{k}) \mid \Phi_{n}\right\rangle$ directly with either numerical integration [17] or CSM [18]. Hence, we propose a new way of obtaining the differential cross section with respect to $\varepsilon$ without calculating the smoothing factor.

Using Eq. (11), one can rewrite the differential cross section as

$$
\begin{aligned}
\frac{d^{2} \sigma}{d \varepsilon d \Omega_{P}} & =\int d \boldsymbol{p}^{\prime} d \boldsymbol{k}^{\prime} \delta\left(\varepsilon-\varepsilon^{\prime}\right)\left|T_{\varepsilon^{\prime}}\left(\boldsymbol{p}^{\prime}, \boldsymbol{k}^{\prime}, \boldsymbol{P}^{\prime}\right)\right|^{2} \\
& \approx \frac{1}{\pi} \mathcal{R}\left(\varepsilon, \Omega_{P}\right),
\end{aligned}
$$

with the generalized response function

$$
\mathcal{R}\left(\varepsilon, \Omega_{P}\right)=\operatorname{Im}\left(\sum_{n, n^{\prime} \neq 0} T_{n}^{*}\left\langle\Phi_{n}\left|G^{(-)}\right| \Phi_{n^{\prime}}\right\rangle T_{n^{\prime}}\right),
$$

where $G^{(-)}=\lim _{\eta \rightarrow+0}\left(\varepsilon-H_{B}-i \eta\right)^{-1}$. In Eq. (14), there is no smoothing factor, as expected. Furthermore, the propagator $G^{(-)}$operates only on spatially damping functions $\Phi_{n}$, so that the calculation of $\left\langle\Phi_{n}\left|G^{(-)}\right| \Phi_{n^{\prime}}\right\rangle$ becomes feasible, as we will show.

CSM is now applied to evaluating $\left\langle\Phi_{n}\left|G^{(-)}\right| \Phi_{n^{\prime}}\right\rangle$. The scaling transformation operator $C(\theta)$ and its inverse are defined by

$$
\begin{aligned}
\langle\boldsymbol{r}, \boldsymbol{y}|C(\theta)| f\rangle & =e^{3 i \theta} f\left(\boldsymbol{r} e^{i \theta}, \boldsymbol{y} e^{i \theta}\right), \\
\left\langle f\left|C^{-1}(\theta)\right| \boldsymbol{r}, \boldsymbol{y}\right\rangle & =\left[e^{-3 i \theta} f\left(\boldsymbol{r} e^{-i \theta}, \boldsymbol{y} e^{-i \theta}\right)\right]^{*} .
\end{aligned}
$$

Using the operators, one can get

$$
\left\langle\Phi_{n}\left|G^{(-)}\right| \Phi_{n^{\prime}}\right\rangle=\left\langle\Phi_{n}\left|C^{-1}(\theta) G_{\theta}^{(-)} C(\theta)\right| \Phi_{n^{\prime}}\right\rangle,
$$


where

$$
G_{\theta}^{(-)}=\lim _{\eta \rightarrow+0} \frac{1}{\varepsilon-H_{B}^{\theta}-i \eta}
$$

with $H_{B}^{\theta}=C(\theta) H_{B} C^{-1}(\theta)$. When $-\pi<\theta<0$, the scaled propagator $\left\langle\boldsymbol{\xi}\left|G_{\theta}^{(-)}\right| \boldsymbol{\xi}^{\prime}\right\rangle$ is a damping function of $\boldsymbol{\xi}$ and $\boldsymbol{\xi}^{\prime}$. It should be noted that although the scaling angle in general calculations with CSM has been taken as positive, the angle in the present situation becomes negative since $G^{(-)}$has the incoming boundary condition. Hence, it can be expanded with $L^{2}$-type basis functions with high accuracy:

$$
G_{\theta}^{(-)} \approx \sum_{i} \frac{\left|\phi_{i}^{\theta}\right\rangle\left\langle\tilde{\phi}_{i}^{\theta}\right|}{\varepsilon-\varepsilon_{i}^{\theta}},
$$

where $\phi_{i}^{\theta}$ is an $i$ th eigenstate obtained by diagonalizing $H_{B}^{\theta}$ in a model space spanned by $L^{2}$-type basis functions:

$$
\left\langle\tilde{\phi}_{i}^{\theta}\left|H_{B}^{\theta}\right| \phi_{i^{\prime}}^{\theta}\right\rangle=\varepsilon_{i}^{\theta} \delta_{i i^{\prime}}
$$

By virtue of CSM, thus, we do not need to evaluate the exact three-body continuum state $\psi_{\varepsilon}^{(-)}(\boldsymbol{k}, \boldsymbol{p})$ to obtain $\left\langle\Phi_{n}\left|G^{(-)}\right| \Phi_{n^{\prime}}\right\rangle$.

Inserting Eq. (19) into Eq. (14) by means of Eq. (17) leads to a useful equation:

$$
\frac{d^{2} \sigma}{d \varepsilon d \Omega_{P}} \approx \frac{1}{\pi} \operatorname{Im} \sum_{i} \frac{T_{i}^{\theta} \tilde{T}_{i}^{\theta}}{\varepsilon-\varepsilon_{i}^{\theta}}
$$

with

$$
\begin{aligned}
\tilde{T}_{i}^{\theta} & \equiv \sum_{n^{\prime}}\left\langle\tilde{\phi}_{i}^{\theta}|C(\theta)| \Phi_{n^{\prime}}\right\rangle T_{n^{\prime}}, \\
T_{i}^{\theta} & \equiv \sum_{n} T_{n}^{*}\left\langle\Phi_{n}\left|C^{-1}(\theta)\right| \phi_{i}^{\theta}\right\rangle .
\end{aligned}
$$

The principal result of this Rapid Communication is that $C(\theta)$ and $C^{-1}(\theta)$ operate only on the spatially damping function $\Phi_{n}$. This makes the calculation of $\left\langle\tilde{\phi}_{i}^{\theta}|C(\theta)| \Phi_{n^{\prime}}\right\rangle$ and $\left\langle\Phi_{n}\left|C^{-1}(\theta)\right| \phi_{i}^{\theta}\right\rangle$ feasible and makes possible the convergence of $d^{2} \sigma / d \varepsilon d \Omega_{P}$ with respect to extending the model space, as we will show. In other words, $C(\theta)$ and $C^{-1}(\theta)$ are not allowed to act on a nondamping function such as the plane wave, since the scaled function diverges asymptotically in this case.

We now test the validity of Eq. (21) for a three-body breakup reaction in which the "exact" breakup $T$-matrix element $T(\boldsymbol{k}, \boldsymbol{P})=\sum_{n}\left\langle\psi^{(-)}(\boldsymbol{k}) \mid \Phi_{n}\right\rangle T_{n}$ is obtainable by taking the overlap $\left\langle\psi^{(-)}(\boldsymbol{k}) \mid \Phi_{n}\right\rangle$ directly with numerical integration. This approach is referred to here as the "smoothing" factor method [12]. As an example, we consider a ${ }^{58} \mathrm{Ni}(d, p n)$ reaction at $80 \mathrm{MeV}$; see Ref. [12] for the details of the CDCC calculation. Figure 1 shows the differential breakup cross section $d \sigma / d \varepsilon$ in which the double differential cross section of Eq. (21) is integrated over the solid angle $\Omega_{P}$ of momentum $\boldsymbol{P}$. The new method (open circles) yields the same result as the smoothing factor method (solid line). Thus, the new method is confirmed to be valid.

Next, the new method is applied to ${ }^{12} \mathrm{C}\left({ }^{6} \mathrm{He}, n n{ }^{4} \mathrm{He}\right)$ scattering at $229.8 \mathrm{MeV}$ as an example of a four-body breakup reaction; see Ref. [16] for optical potentials between $n$ and ${ }^{12} \mathrm{C}$ and between $\alpha$ and ${ }^{12} \mathrm{C}$. As interactions $V_{n n}$ and $V_{n \alpha}$

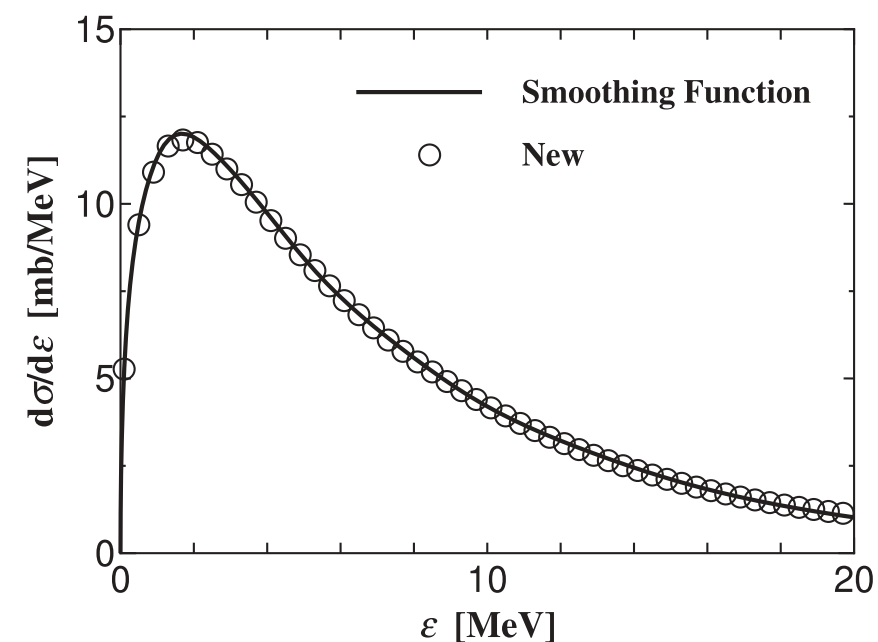

FIG. 1. Calculated breakup cross section for $d+{ }^{58} \mathrm{Ni}$ scattering at $80 \mathrm{MeV}$. The solid line shows the result of the smoothing factor method; open circles show the result using the new method.

in $H_{B}$, we take the so-called GPT [27] and KKNN [28] potentials, respectively. These potentials with a Gaussian form reproduce well data of low-energy nucleon-nucleon and nucleon- ${ }^{4} \mathrm{He}$ scattering, respectively. The particle exchange between valence neutrons and neutrons in ${ }^{4} \mathrm{He}$ is treated approximately with the orthogonality condition model [29].

For the diagonalization of $H_{B}$ and $H_{B}^{\theta}$, we adopt the Gaussian expansion method (GEM) [30]. In GEM, the state of the ${ }^{4} \mathrm{He}+n+n$ system is described by a superposition of three channels, each channel with a different set of Jacobi coordinates $\left(\boldsymbol{y}_{c}, \boldsymbol{r}_{c}\right)$. For each channel $(c)$, the radial parts of the internal wave functions involving $\boldsymbol{y}_{c}$ and $\boldsymbol{r}_{c}$ are expanded by a finite number of Gaussian basis functions

$$
\begin{aligned}
\varphi_{j \lambda}\left(\boldsymbol{y}_{c}\right) & =y_{c}^{\lambda} e^{-\left(y_{c} / \bar{y}_{j}\right)^{2}} Y_{\lambda}\left(\Omega_{y_{c}}\right), \\
\varphi_{i \ell}\left(\boldsymbol{r}_{c}\right) & =r_{c}^{\ell} e^{-\left(r_{c} / \bar{r}_{i}\right)^{2}} Y_{\ell}\left(\Omega_{r_{c}}\right),
\end{aligned}
$$

respectively. Here $\lambda(\ell)$ is the angular momentum of $\boldsymbol{y}_{c}\left(\boldsymbol{r}_{c}\right)$, and the range parameters are taken to lie in geometric progression:

$$
\begin{aligned}
\bar{y}_{j} & =\left(\bar{y}_{\max } / \bar{y}_{1}\right)^{(j-1) / j_{\max }}, \\
\bar{r}_{i} & =\left(\bar{r}_{\max } / \bar{r}_{1}\right)^{(i-1) / i_{\max }} .
\end{aligned}
$$

The parameters depend on $c$, but we omitted the dependence in Eqs. (25) and (26) for simplicity; see Ref. [14] for the details of the diagonalization and the definition of Jacobi coordinates.

In order to confirm the convergence of the breakup cross section with respect to extending the model space, we prepared three sets of basis functions, shown in Table I. For $0^{+}$and $1^{-}$states, maximum internal angular momenta are set to $\ell=\lambda=1$. For $2^{+}$states, they are $\ell=\lambda=1$ for $c=1$ and 2 and $\ell=\lambda=2$ for $c=3$.

Figure 2 shows the breakup cross sections $d \sigma / d \varepsilon$ to $0^{+}$ and $1^{-}$continua separately, and Fig. 3 shows the cross section to the $2^{+}$continuum. For all the cross sections, sets II and III yield the same result, but the result of set I is somewhat different from those of sets II and III. Thus, the convergence with respect to increasing the model space is obtained with set II. Figure 4 shows the $\theta$ dependence of the net breakup 
TABLE I. Gaussian range parameters.

\begin{tabular}{lccccccc}
\hline \hline Set & $c$ & $j_{\max }$ & $\bar{y}_{1}(\mathrm{fm})$ & $\bar{y}_{\max }(\mathrm{fm})$ & $i_{\max }$ & $\bar{r}_{1}(\mathrm{fm})$ & $\bar{r}_{\max }(\mathrm{fm})$ \\
\hline I & 3 & 10 & 0.1 & 10.0 & 10 & 0.5 & 10.0 \\
& 1,2 & 10 & 0.5 & 10.0 & 10 & 0.5 & 10.0 \\
II & 3 & 15 & 0.1 & 20.0 & 15 & 0.5 & 20.0 \\
& 1,2 & 15 & 0.5 & 20.0 & 15 & 0.5 & 20.0 \\
III & 3 & 20 & 0.1 & 50.0 & 20 & 0.5 & 50.0 \\
& 1,2 & 20 & 0.5 & 50.0 & 20 & 0.5 & 50.0 \\
\hline \hline
\end{tabular}

cross section to $0^{+}, 1^{-}$, and $2^{+}$continua around the $2^{+}$ resonance peak. The net breakup cross section converges at $\theta=-14^{\circ}$ when $\theta$ decreases from $-6^{\circ}$ to $-18^{\circ}$.

The present calculation includes Coulomb breakup processes that were neglected in the previous paper [15]. The effect is significant for the $1^{-}$continuum, as shown by the dotted line in Fig. 2(b). It enhances the breakup cross section by a factor of 2 from the result without Coulomb breakup processes. The effect is negligible for $0^{+}$and $2^{+}$continua, although the latter is a dominant component of the breakup cross section. Consequently, the Coulomb breakup effect in the present reaction system is not significant for either the breakup reaction or elastic scattering. For heavy targets, Coulomb breakup processes dominate breakup reactions, and Coulomb breakup reactions are a useful tool for investigating properties of halo nuclei. The new method proposed here can treat both

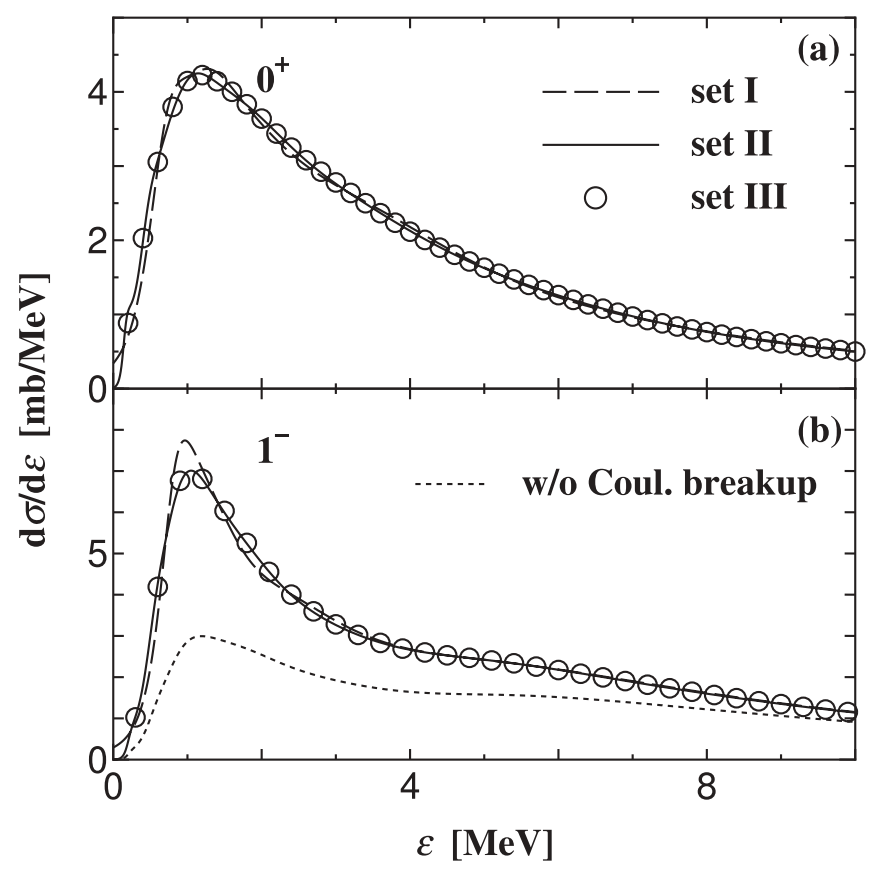

FIG. 2. Convergence of the breakup cross sections to the (a) $0^{+}$ continuum and (b) $1^{-}$continuum. Dashed lines, solid lines, and open circles correspond to results of sets I, II, and III, respectively. The dotted line in (b) shows the results when Coulomb breakup processes are switched off.

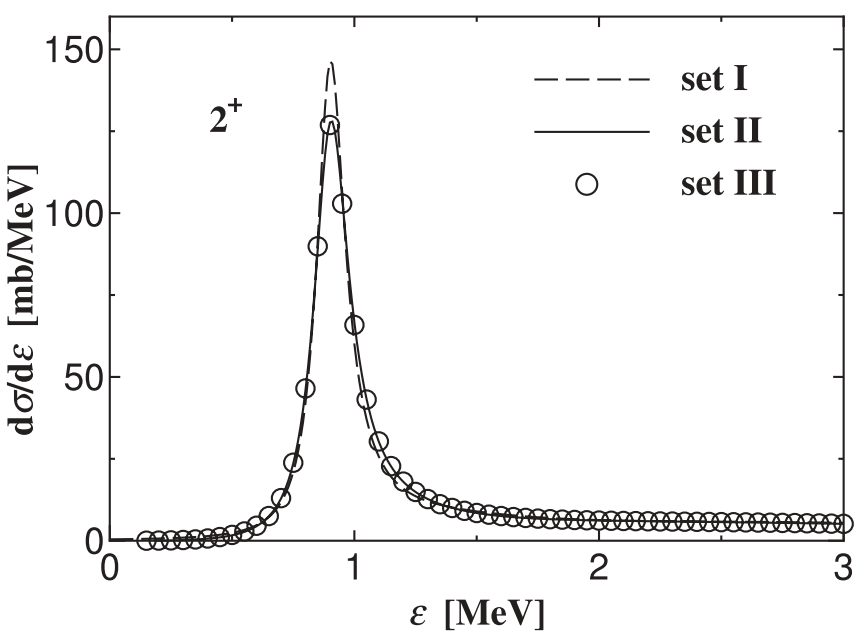

FIG. 3. Convergence of the breakup cross sections to the $2^{+}$ continuum. Definition of lines is the same as in Fig. 2.

nuclear and Coulomb breakup processes and then be used to analyze their interference in the same framework.

In Fig. 5, the breakup cross section $d \sigma / d \varepsilon$ calculated by the present method is compared with the experimental data for ${ }^{6} \mathrm{He}+{ }^{12} \mathrm{C}$ and ${ }^{6} \mathrm{He}+{ }^{208} \mathrm{~Pb}$ reactions at $240 \mathrm{MeV} / A$ [31]. These data have already been analyzed by a four-body distorted-wave Born approximation (DWBA) [32] and the eikonal approximation [33]. In the present calculation, we take the same potentials as in Ref. [33] for $n-{ }^{208} \mathrm{~Pb}$ and $\alpha-{ }^{208} \mathrm{~Pb}$ systems. The optical potential for a $n-{ }^{12} \mathrm{C}$ system is taken from the global nucleon-nucleus potential [34], while the optical potential for $\alpha{ }^{12} \mathrm{C}$ system is constructed from the ${ }^{12} \mathrm{C}+{ }^{12} \mathrm{C}$ potential at $200 \mathrm{MeV} / A$ [35] by changing the radius parameter from ${ }^{12} \mathrm{C}$ to $\alpha$. Nuclear breakup is dominant for ${ }^{6} \mathrm{He}+{ }^{12} \mathrm{C}$ scattering at $240 \mathrm{MeV} / A$, while Coulomb breakup to the $1^{-}$ continuum is dominant for ${ }^{6} \mathrm{He}+{ }^{208} \mathrm{~Pb}$ scattering. For a ${ }^{12} \mathrm{C}$ target, the present theoretical result is consistent with the experimental data except for the peak of the $2^{+}$resonance around $\varepsilon=1 \mathrm{MeV}$. This overestimation is also seen in the results of four-body DWBA, and the problem is partly solved by

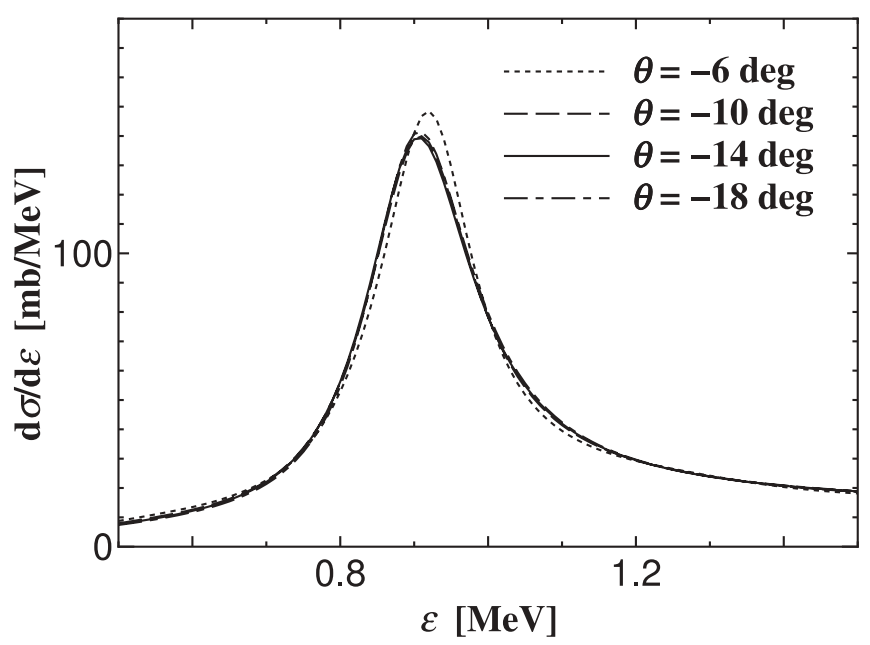

FIG. 4. Dependence of the net breakup cross section to $0^{+}, 1^{-}$, and $2^{+}$continua on $\theta$. 


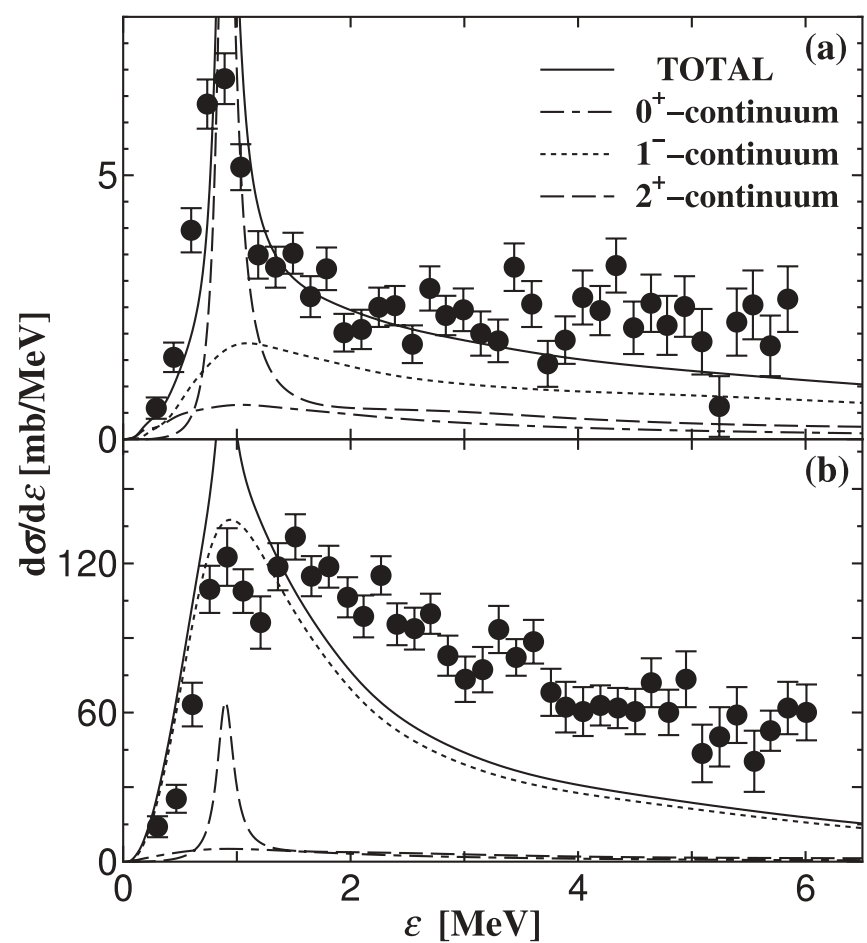

FIG. 5. Comparison of the breakup cross section calculated by CDCC (solid line) with experimental data for (a) ${ }^{6} \mathrm{He}+{ }^{12} \mathrm{C}$ scattering at $240 \mathrm{MeV} / A$ and (b) ${ }^{6} \mathrm{He}+{ }^{208} \mathrm{~Pb}$ scattering at $240 \mathrm{MeV} / A$. Dotdashed, dotted, and dashed lines show contributions of $0^{+}, 1^{-}$, and $2^{+}$breakup, respectively. Experimental data are taken from Ref. [31]. considering the experimental energy resolution. For a ${ }^{208} \mathrm{~Pb}$ target, the present method underestimates the experimental data at $\varepsilon \gtrsim 2 \mathrm{MeV}$. A possible origin of this underestimation is that the inelastic breakup reactions are not included in the present calculation. As mentioned in Ref. [32], the inelastic breakup effect is not negligible, and the elastic breakup cross section calculated with four-body DWBA also underestimates the data.

In summary, we have proposed a practical method of calculating the differential breakup cross section as a continuous function of the excitation energy of a projectile by combining $\mathrm{CDCC}$ and CSM. This method does not require one to calculate the continuum wave functions of the projectile. All we have to do is just diagonalize the projectile Hamiltonian and the scaled Hamiltonian with $L^{2}$-type basis functions. In the present formalism, the scaling operator $C(\theta)$ operates only on spatially damping functions, and hence the differential breakup cross section converges quickly as the model space is extended. The method is successful in reproducing the data for ${ }^{6} \mathrm{He}+{ }^{12} \mathrm{C}$ and ${ }^{6} \mathrm{He}+{ }^{208} \mathrm{~Pb}$ reactions at $240 \mathrm{MeV} / A$. In principle, the present formalism is applicable for the many-body breakup reaction if the diagonalization of the projectile Hamiltonian and the scaled Hamiltonian is feasible.

The authors would like to thank Dr. K. Ogata for useful and helpful discussions. M.Y. is grateful to M. Rodríguez-Gallardo and A. M. Moro for discussions and suggestions at DREB2009. The numerical calculations of this work were performed on the computing system at the Research Institute for Information Technology of Kyushu University.
[1] I. Tanihata et al., Phys. Lett. B 289, 261 (1992).

[2] I. Tanihata, J. Phys. G 22, 157 (1996).

[3] P. G. Hansen et al., Annu. Rev. Nucl. Part. Sci. 45, 591 (1995).

[4] E. K. Warburton, J. A. Becker, and B. A. Brown, Phys. Rev. C 41, 1147 (1990).

[5] M. Kamimura et al., Prog. Theor. Phys. Suppl. 89, 1 (1986).

[6] N. Austern et al., Phys. Rep. 154, 125 (1987).

[7] M. Yahiro et al., Prog. Theor. Phys. 67, 1467 (1982).

[8] R. A. D. Piyadasa et al., Prog. Theor. Phys. 81, 910 (1989).

[9] N. Austern, M. Yahiro, and M. Kawai, Phys. Rev. Lett. 63, 2649 (1989).

[10] N. Austern, M. Kawai, and M. Yahiro, Phys. Rev. C 53, 314 (1996).

[11] A. M. Moro et al., Phys. Rev. C 65, 011602(R) (2001).

[12] T. Matsumoto et al., Phys. Rev. C 68, 064607 (2003).

[13] T. Egami et al., Phys. Rev. C 70, 047604 (2004).

[14] T. Matsumoto et al., Phys. Rev. C 70, 061601(R) (2004).

[15] T. Matsumoto et al., Phys. Rev. C 73, 051602(R) (2006).

[16] M. Rodríguez-Gallardo et al., Phys. Rev. C 77, 064609 (2008).

[17] T. Egami et al., Prog. Theor. Phys. 121, 789 (2009).

[18] T. Matsumoto et al., Prog. Theor. Phys. 121, 885 (2009).
[19] J. Aguilar and J. M. Combes, Commun. Math. Phys. 22, 269 (1971); E. Balslev and J. M. Combes, ibid. 22, 280 (1971).

[20] I. J. Thompson et al., Comput. Phys. Commun. 161, 87 (2004).

[21] M. Rodríguez-Gallardo et al., Phys. Rev. C 80, 051601(R) (2009).

[22] S. Aoyama et al., Prog. Theor. Phys. 116, 1 (2006).

[23] T. Myo, K. Kato, S. Aoyama, and K. Ikeda, Phys. Rev. C 63, 054313 (2001).

[24] T. Myo, K. Kato, H. Toki, and K. Ikeda, Phys. Rev. C 76, 024305 (2007).

[25] B. V. Danilin et al., Phys. Rev. C 55, R577 (1997).

[26] B. V. Danilin et al., Nucl. Phys. A 632, 383 (1998).

[27] D. Gogny et al., Phys. Lett. B 32, 591 (1970).

[28] H. Kanada et al., Prog. Theor. Phys. 61, 1327 (1979).

[29] S. Saito, Prog. Theor. Phys. 41, 705 (1969).

[30] E. Hiyama et al., Prog. Part. Nucl. Phys. 51, 223 (2003).

[31] T. Aumann et al., Phys. Rev. C 59, 1252 (1999).

[32] S. N. Ershov, B. V. Danilin, and J. S. Vaagen, Phys. Rev. C 62, 041001(R) (2000).

[33] D. Baye, P. Capel, P. Descouvemont, and Y. Suzuki, Phys. Rev. C 79, 024607 (2009).

[34] A. J. Koning and J. P. Delaroche, Nucl. Phys. A 713, 231 (2003).

[35] J. Y. Hostachy et al., Nucl. Phys. A 490, 441 (1988). 